\title{
ON THE STRENGTH OF WEAK COMPACTNESS
}

\author{
ALEXANDER P. KREUZER
}

\begin{abstract}
We study the logical and computational strength of weak compactness in the separable Hilbert space $\ell_{2}$.

Let weak-BW be the statement the every bounded sequence in $\ell_{2}$ has a weak cluster point. It is known that weak-BW is equivalent to $\mathrm{ACA}_{0}$ over $\mathrm{RCA}$ and thus that it is equivalent to (nested uses of) the usual Bolzano-Weierstraß principle BW.

We show that weak-BW is instance-wise equivalent to the $\Pi_{2}^{0}$-CA. This means that for each $\Pi_{2}^{0}$ sentence $\mathrm{A}(n)$ there is a sequence $\left(x_{i}\right)_{i \in \mathbb{N}}$ in $\ell_{2}$, such that one can define the comprehension functions for $\mathrm{A}(n)$ recursively in a cluster point of $\left(x_{i}\right)_{i}$. As consequence we obtain that the degrees $d \geq_{T} 0^{\prime \prime}$ are exactly those degrees that contain a weak cluster point of any computable, bounded sequence in $\ell_{2}$. Since a cluster point of any sequence in the unit interval $[0,1]$ can be computed in a degree low over $0^{\prime}$ (see [10]), this show also that instances of weak-BW are strictly stronger than instances of BW.

We also comment on the strength of weak-BW in the context of abstract Hilbert spaces in the sense of Kohlenbach and show that his construction of a solution for the functional interpretation of weak compactness is optimal, cf. [7.
\end{abstract}

We investigate the computational and logical strength of weak sequential compactness in the separable Hilbert space $\ell_{2}$.

The strength of weak compactness has so far only been studied in the context of proof mining, see [9, 7]. There general Hilbert spaces in a more general logical system are considered. It is straightforward to deduce from this analysis that weak compactness for $\ell_{2}$ is equivalent to $\mathrm{ACA}_{0}$ over $\mathrm{RCA}$.

In this paper we refine this result and show that weak compactness on $\ell_{2}$ is instance-wise equivalent to $\Pi_{2}^{0}-\mathrm{CA}$ over $\mathrm{RCA}$. This means that for each bounded sequence in $\ell_{2}$ one can uniformly compute a function $f$ such that from a comprehension function for $\forall x \exists y f(x, y, n)=0$ one can compute a weak cluster point and vice versa.

As consequence we obtain that the degrees $d \geq_{T} 0^{\prime \prime}$ are exactly those degrees that compute a weak cluster point for each computable bounded sequence in $\ell_{2}$ and that there is a computable bounded sequence in $\ell_{2}$ such that from a cluster point of this sequence one can compute $0^{\prime \prime}$.

This shows that instances of the Bolzano-Weierstraß principle for weak compactness are strictly stronger than instances of the usual Bolzano-Weierstraß principle.

2010 Mathematics Subject Classification. Primary 03F60; Secondary 03D80, 03B30.

Key words and phrases. Bolzano-Weierstraß principle, weak sequential compactness, Turing degree, abstract Hilbert space.

The author is supported by the German Science Foundation (DFG Project KO 1737/5-1).

I am grateful to Ulrich Kohlenbach for useful discussions and suggestions for improving the presentation of the material in this article. 
This paper is organized as follows: first the Hilbert space $\ell_{2}$ is defined. This definition follows 11, 1]. Then the actual results are proven (Theorems 8 and 12) and we show that the result can also be formulated for abstract Hilbert spaces, in the sense of Kohlenbach 8 (Theorem 10). As corollary of this we obtain that Kohlenbach's analysis of the weak compactness functional $\Omega^{*}$ in $[7$ is optimal (Corollary 11). At the end, we reformulate the result of the analysis in terms of the Weihrauch lattice (Remark 14).

Definition 1 (vector space, [11, II.10]). A countable vector space A over a countable field $K$ consists of a set $|A| \subseteq \mathbb{N}$ with operations $+:|A| \times|A| \rightarrow|A|$ and $\cdot:|K| \times$ $|A| \rightarrow|A|$ and a distinguished element $0 \in|A|$ such that $(|A|,+, \cdot, 0)$ satisfies the usual axioms for a vector space over $K$.

Definition 2 (Hilbert space, 1, Definition 9.3]). A (real) separable Hilbert space $H$ consists of a countable vector space $A$ over $\mathbb{Q}$ together with a function $\langle\cdot, \cdot\rangle: A \times A \rightarrow$ $\mathbb{R}$ satisfying

(1) $\langle x, x\rangle \geq 0$,

(2) $\langle x, y\rangle=\langle y, x\rangle$,

(3) $\langle a x+b y, z\rangle=a\langle x, z\rangle+b\langle y, z\rangle$,

for all $x, y, z \in A$ and $a, b \in \mathbb{Q}$.

The inner product on $H$ induces a pseudonorm $\|x\|:=\sqrt{\langle x, x\rangle}$. We think of the Hilbert space $H$ as the completion of $A$ under the pseudometric $d(x, y)=\|x-y\|$. Thus an element of $H$ consists of a sequence $\left(x_{n}\right)_{n \in \mathbb{N}} \subseteq A$, such that $d\left(x_{n}, x_{m}\right)<$ $2^{-n}$ for all $m>n$. The inner product $\langle\cdot, \cdot\rangle$ is continuously extended to the whole space $H$.

A Hilbert space is finite dimensional if it is spanned by finitely many vectors. If this is not the case we say that it is infinite dimensional.

Avigad, Simic showed in [1, Theorem 10.9] that every Hilbert space $H$ in the sense of Definition 2 has a orthonormal basis. Since each such Hilbert space is separable this basis is at most countable.

As consequence of this each two infinite dimensional (separable) Hilbert spaces are isomorphic over $\mathrm{RCA}_{0}$, see [1, Corollary 10.11]. Thus we many restrict our attention to $\ell_{2}$, as given by the following definition.

Definition $3\left(\ell_{2}\right.$, [11, II.10.2]). Let $A=(|A|,+, \cdot, 0)$ be a vector space over $\mathbb{Q}$, where $|A|$ is the set of all finite sequences of rational numbers $\left\langle r_{0}, \ldots, r_{m}\right\rangle$, such that either $m=0$ or $r_{m} \neq 0$. Addition is defined by putting $\left\langle r_{0}, \ldots, r_{m}\right\rangle+\left\langle s_{0}, \ldots, s_{n}\right\rangle=$ $\left\langle r_{0}+s_{0}, \ldots, r_{k}+s_{k}\right\rangle$ where $r_{i}, s_{i}=0$ for $i>m, n$ and $k=\max \left\{i \mid i=0 \vee r_{i}+s_{i} \neq\right.$ $0\}$. For scalar multiplication put $q \cdot\left\langle r_{0}, \ldots, r_{m}\right\rangle=\langle 0\rangle$ if $q=0$ and $\left\langle q \cdot r_{0}, \ldots, q \cdot r_{m}\right\rangle$ otherwise.

The space $\ell_{2}$ is defined to be the Hilbert space consisting of $A$ with the inner product

$$
\left\langle\left\langle r_{0}, \ldots, r_{m}\right\rangle,\left\langle s_{0}, \ldots, s_{n}\right\rangle\right\rangle=\sum_{i=0}^{\max (n, m)} r_{i} s_{i}
$$

The canonical orthonormal basis $\left(e_{n}\right)_{n}$ of $\ell_{2}$ is given by

$$
e_{n}=\langle\underbrace{0, \ldots, 0}_{n \text { times }}, 1\rangle \text {. }
$$


Definition 4 (projection). Let $M$ be a closed linear subspace of a Hilbert space $H$. A point $y \in M$ is called projection of $x \in H$ if $x-y$ is orthogonal to (each element of) $M$.

A bounded linear operator $P_{M}$ on $H$ that maps each point of $H$ to its projection on $M$ is called projection function for $M$.

Usually projections are defined differently, see e.g. 1, Definition 12.1]. Avigad, Simic showed that this definition is over $\mathrm{RCA}_{0}$ equivalent to the usual definition, see [1, Lemma 12.2].

We immediately obtain the following lemma:

Lemma 5. Let $N \subset \mathbb{N}$ and $M$ be the subspace of $\ell_{2}$ that is spanned by $\left\{e_{n} \mid n \in N\right\}$. Then $\mathrm{RCA}_{0}$ proves that the projection $P_{M}$ of $\ell_{2}$ onto the space $M$ exists.

Proof. The projection of an element $\left\langle r_{0}, \ldots, r_{m}\right\rangle$ of the space $|A|$ is given by $\left\langle r_{0}^{\prime}, \ldots, r_{m^{\prime}}^{\prime}\right\rangle$, where $r_{i}^{\prime}=r_{i}$ if $n \in N$ and $r_{i}^{\prime}=0$ if $n \notin N$ and $m^{\prime}=\max \left\{i \leq m \mid r_{i} \neq 0 \vee i=0\right\}$.

It is easy to show that $P_{M}$ is linear and that is bounded by 1 (at least on $|A|$ ). From this one can deduce that $P_{M}$ is continuous and continuously extend it to the full space $\ell_{2}$.

Definition 6 (weak convergence). We say that a sequence $\left(x_{i}\right)_{i \in \mathbb{N}}$ of elements of a Hilbert space $H$ converges weakly to a point $x$ if

$$
\forall y \in H \lim _{i \rightarrow \infty}\left\langle y, x_{i}\right\rangle=\langle y, x\rangle .
$$

The Bolzano-Weierstraß principle for weak convergence is defined to be the statement that for every bounded sequence $\left(x_{i}\right)_{i \in \mathbb{N}}$ of elements of $H$ there exists a point $x$ such that a subsequence of $\left(x_{i}\right)_{i}$ converges weakly to $x$. This principle is abbreviated by weak-BW. The restriction of this principle to a fixed sequence $\left(x_{i}\right)_{i \in \mathbb{N}}$ is denoted by weak-BW $\left(\left(x_{i}\right)_{i}\right)$.

If $H$ has an orthonormal basis it is sufficient to have (10) only for all $y$ in the basis.

Lemma 7. Projections are weakly continuous in the sense that if $x$ is the weak limit point of a sequence $\left(x_{i}\right)_{i \in \mathbb{N}}$, then $P x$ is the weak limit point of $\left(P x_{i}\right)_{i \in \mathbb{N}}$ for any projection $P$.

Proof. Follows from the definition of the projection and the continuity of $\langle\cdot, \cdot\rangle$.

An instance of $\Pi_{2}^{0}$-comprehension given by a $\Pi_{2}^{0}$ formula $\mathrm{A}(n)$ is the statement

$$
\exists g \forall n(g(n)=0 \leftrightarrow \mathrm{A}(n)) .
$$

Since all $\Pi_{2}^{0}$-formulas $\mathrm{A}(n)$ can be written as $\forall x \exists y \operatorname{txyn}=0$ for a (primitive recursive) term $t$, we can rewrite this as

$$
\exists g \forall n(g(n)=0 \leftrightarrow \forall x \exists y \operatorname{txyn}=0) .
$$

This statement will be abbreviated by $\Pi_{2}^{0}-\mathrm{CA}(t)$. (In order to be able to formulate this in $\mathrm{RCA}_{0}$ we will use the conservative extension by all primitive recursive functions of it.)

Theorem 8. For each instance $\mathrm{A}(n) \equiv[\forall x \exists y$ txyn $=0]$ of $\Pi_{2}^{0}$-CA there exists a bounded sequence $\left(x_{i}\right)_{i \in \mathbb{N}}$ in $\ell_{2}$, such that

$$
\mathrm{RCA}_{0} \vdash \text { weak-BW }\left(\left(x_{i}\right)_{i \in \mathbb{N}}\right) \rightarrow \Pi_{2}^{0}-\mathrm{CA}(t) .
$$


Moreover, the sequence $\left(x_{i}\right)_{i \in \mathbb{N}}$ can be primitive recursively and uniformly computed from $t$, i.e. there is a primitive recursive functional $F$ such that $x_{i}=F(t, i)$.

Proof. Define

$$
f(n, i):=\max \left\{x \leq i \mid \forall x^{\prime}<x \exists y<i\left(t\left(x^{\prime}, y, n\right)=0\right)\right\} .
$$

It is clear that $\lambda i . f(n, i)$ is increasing for each $n$.

Claim 1.

$$
\mathrm{A}(n) \quad \text { iff } \quad \lambda i . f(n, i) \text { is unbounded, i.e. } \forall k \exists i(f(n, i)>k) .
$$

Proof of Claim 1.

- The right to left direction follows immediately from the definition of $f$.

- For the left to right direction fix an $n$. We will show that not the right side implies not the left side.

Hence assume that $\lambda i . f(n, i)$ is bounded by $k$, i.e.

$$
\forall i(f(n, i) \leq k) .
$$

By $\Sigma_{1}^{0}$-induction we may assume that $k$ is minimal and thus

$$
\exists i(f(n, i)=k) .
$$

From the definition of $f$ we obtain

$$
\forall x<k \exists y(t(x, y, n)=0) .
$$

Together with (2) we obtain that

$$
\forall y(t(k, y, n) \neq 0)
$$

and hence $\neg \mathrm{A}(n)$.

This proofs the claim.

Let

$$
y_{n, i}:=e_{\langle n, f(n, i)\rangle} .
$$

The sequence $\left(y_{n, i}\right)_{i \in \mathbb{N}}$ is obviously bounded by 1 and hence possesses for each $n$ a weak cluster point $y_{n}$.

Claim 2.

- $\left\|y_{n}\right\|=_{\mathbb{R}} 0$, if $\mathrm{A}(n)$ and

- $\left\|y_{n}\right\|=_{\mathbb{R}} 1$, if $\neg \mathrm{A}(n)$.

Proof of Claim 2.

- If $\mathrm{A}(n)$ is true, then $\lambda i . f(n, i)$ is unbounded and hence $\left\langle e_{j}, y_{n, i}\right\rangle$ eventually becomes 0 . Therefore $y_{n, i}$ converges weakly to 0 .

- If $\mathrm{A}(n)$ is false, then $\lambda i . f(n, i)$ is bounded. By $\Sigma_{1}^{0}$-induction we obtain a smallest upper bound $k$ and since $\lambda i . f(n, i)$ is increasing we obtain that $\lim _{i \rightarrow \infty} f(n, i)=k$. As consequence we obtain that $y_{n, i}$ eventually becomes constant $e_{\langle n, k\rangle}$ and hence that $y_{n}=e_{\langle n, k\rangle}$ and $\left\|y_{n}\right\|={ }_{\mathbb{R}} 1$.

This proves the claim.

We parallelize this process to obtain the comprehension function for $\mathrm{A}(n)$. For this let

$$
x_{i}:=\sum_{n=0}^{i} 2^{-\frac{n+1}{2}} y_{n, i} .
$$


Since the $y_{n, i}$ are orthogonal for different $n$, we obtain by Pythagoras that

$$
\left\|x_{i}\right\|^{2}=\sum_{n=0}^{i} 2^{-(n+1)}\left\|y_{n, i}\right\|^{2} \leq 1
$$

and thus that $\left(x_{i}\right)$ is bounded.

By weak-BW $\left(\left(x_{i}\right)_{i}\right)$ there exists a weak cluster point $x$ of $\left(x_{i}\right)$. Let now $M_{n}$ be the closed linear space spanned by $\left\{e_{\langle n, k\rangle} \mid k \in \mathbb{N}\right\}$. By definition the subspaces $M_{n}$ are disjoint (except for the 0 vector) for different $n$, and $y_{n, i} \in M_{n}$ for all $i, n$.

By Lemma 5 the projections $P_{M_{n}}$ onto the spaces $M_{n}$ exist. For this projections we have

$$
P_{M_{n}}\left(x_{i}\right)=2^{-\frac{n+1}{2}} y_{n, i} \text { for } n \geq i .
$$

Since $P_{M_{n}}$ is weakly continuous, see Lemma 7 we get

$$
P_{M_{n}}(x)=2^{-\frac{n+1}{2}} y_{n}
$$

Now Claim 2 yields that $\left\|P_{M_{n}}(x)\right\|==_{\mathbb{R}} 0$ if $\mathrm{A}(n)$ and $\left\|P_{M_{n}}(x)\right\|==_{\mathbb{R}} 2^{-\frac{n+1}{2}}$ if $\neg \mathrm{A}(n)$. Hence the function

$$
g(n):= \begin{cases}0 & \text { if }\left\|P_{M_{n}}(x)\right\|(n+1)<\mathbb{Q} 2^{-\frac{n+1}{2}}, \\ 1 & \text { otherwise }\end{cases}
$$

where $\left\|P_{M_{n}}(x)\right\|(n+1)$ is a $2^{-(n+1)}$ good rational approximation of $\left\|P_{M_{n}}(x)\right\|$, provides a comprehension function and solves the theorem.

It is clear that $\left(x_{i}\right)$ is primitive recursive in $t$.

As immediate consequence we obtain the following corollary:

Corollary 9. There is a sequence $\left(x_{i}\right)_{i}$ of elements in $\ell_{2}$ such that from a cluster point $x$ of this sequence one can compute any element of the second Turing jump $0^{\prime \prime}$.

Proof. Take for $\mathrm{A}(n)$ in Theorem 8 the $\Pi_{2}^{0}$ statement that the Turing machine $\{n\}^{0^{\prime}}(n)$ halts.

Kohlenbach studies weak compactness in the context of arbitrary abstract Hilbert spaces, see 8, 9. By abstract Hilbert space we mean that the Hilbert space is added as a new type to the system together with the Hilbert space axioms and that the space is not coded as sequences of numbers. With this one can analyze Hilbert spaces without referring to a concrete space like $\ell_{2}$ and one does not automatically obtain a separable Hilbert space but can analyze general Hilbert spaces.

We do not introduce the notation for abstract Hilbert spaces here but refer the reader to [8, Chapter 17]. We show now that the statement of Theorem 8 is also applicable in this context:

Theorem 10. Let $\widehat{\mathrm{PA}}^{\omega} \uparrow[X,\langle\cdot, \cdot\rangle]$ be the extension of $\widehat{\mathrm{PA}}^{\omega} \uparrow$ by the abstract Hilbert space $X$ with the scalar product $\langle\cdot, \cdot\rangle$ and let now weak-BW denote the BolzanoWeierstraß principle for weak compactness in $X$. Then

$$
\begin{aligned}
\widehat{\mathrm{PA}}^{\omega} \uparrow[X,\langle\cdot, \cdot\rangle]+\Pi_{1}^{0}-\mathrm{CP} \vdash\left(\exists\left(e_{i}\right)_{i \in \mathbb{N}}\right. & \left.\forall i, j\left\langle e_{i}, e_{j}\right\rangle=\delta_{i j}\right) \\
& \rightarrow \exists\left(x_{i}\right)_{i \in \mathbb{N}}\left(\text { weak-BW }\left(\left(x_{i}\right)_{i}\right) \rightarrow \Pi_{2}^{0}-\mathrm{CA}(t)\right) .
\end{aligned}
$$


In other words, if $X$ is provably infinite dimensional, then Theorem 8 also holds with $\ell_{2}$ replaced by $X$.

Proof. The only step in the proof of Theorem 8 that does not formalize in $\widehat{\mathrm{PA}}^{\omega} \uparrow[\mathrm{X},\langle\cdot, \cdot\rangle]$ is projection of $x$ onto $M_{n}$, i.e. Lemma 5 since this depends on the coding of $\ell_{2}$.

We show now how to obtain this projection of $x$ in this system. For this consider

$$
\begin{aligned}
\|x\|^{2} & =\langle x, x\rangle=\lim _{i \rightarrow \infty}\left\langle x, x_{i}\right\rangle \\
& =\lim _{i \rightarrow \infty} \sum_{n=0}^{i} 2^{-(n+1)}\left\langle x, y_{n, i}\right\rangle \\
& \leq \lim _{i \rightarrow \infty} \sum_{n=0}^{k} 2^{-(n+1)}\left\langle x, y_{n, i}\right\rangle+2^{-k} \quad \text { for each } k \\
& =\sum_{n=0}^{k} 2^{-(n+1)} \lim _{i \rightarrow \infty}\left\langle x, y_{n, i}\right\rangle+2^{-k} .
\end{aligned}
$$

Now

$$
\left\langle x, y_{n, i}\right\rangle=\lim _{j \rightarrow \infty}\left\langle x_{j}, y_{n, i}\right\rangle=2^{-(n+1)} \lim _{j \rightarrow \infty}\left\langle y_{n, j}, y_{n, i}\right\rangle .
$$

Thus, by the definition of $y_{n, i}$ the term $\left\langle x, y_{n, i}\right\rangle$ is monotone in $i$ and in particular for each $n$ there is an $m$, such that

$$
\lim _{i \rightarrow \infty}\left\langle x, y_{n, i}\right\rangle=\left\langle x, y_{n, i^{\prime}}\right\rangle \quad \text { for } i^{\prime} \geq m .
$$

By $\Pi_{1}^{0}$-CP there is now an $m$ which does it for all $n \leq k$. Hence, we obtain

$$
\forall k \exists i\|x\|^{2} \leq \sum_{n=0}^{k} 2^{-(n+1)}\left\langle x, y_{n, i}\right\rangle+2^{-k} .
$$

By (3) the term $\left\langle x, y_{n, i}\right\rangle$ is either 0 or $2^{-(n+1)}$, hence

$$
=\sum_{n=0}^{k}\left\langle x, y_{n, i}\right\rangle^{2}+2^{-k}
$$

Thus, $\sum_{n=0}^{k}\left\langle x, y_{n, i}\right\rangle y_{n, i}$ is a $2^{-k / 2} \operatorname{good}$ approximation of $x$ consisting of finite linear combinations of $\left(e_{i}\right)$. Using an application of QF-AC one easily obtains a sequence of approximations converging to $x$ at the rate $2^{-k}$. Using this one can obtain $P_{M_{n}}(x)$ like in Lemma 5

This proves the theorem.

By applying the functional interpretation to this we obtain the following corollary:

Corollary 11. Let $\Omega$ be a solution of the functional interpretation of weak-BW then for every $n \geq 1$ there are terms in $T_{n}$, such that the application of $\Omega$ to these terms is (extensionally) equal to a function definable in the $T_{n+2}$ but not in $T_{n+1}$.

Proof. Let A be the statement that the function $f_{\omega_{n+1}}$ from the fast growing hierarchy is total. It is well known that the statement $A$ cannot be proven in $\Sigma_{n+2}^{0}$-IA but can be proven using a suitable instance of $\Sigma_{n+3}^{0}$ IA, see [5, II.3.(d)]. Thus a 
solution of the functional interpretation of A cannot be found in $T_{n+1}$ but can be found in $T_{n+2}$.

Let $\widehat{\mathrm{PA}}^{\omega} \uparrow\left[X,\langle\cdot, \cdot\rangle,\left(e_{i}\right)_{i \in \mathbb{N}}\right]$ be the extension of $\widehat{\mathrm{PA}}^{\omega} \uparrow[X,\langle\cdot, \cdot\rangle]$ by the constant $\left(e_{i}\right)_{i}$, which can be majorized by $\lambda i .1$, and the axiom $\forall i, j \in \mathbb{N}\left\langle e_{i}, e_{j}\right\rangle=\mathbb{R} \delta_{i j}$. For this system the metatheorem [8, Theorem 17.69.2)], see also [4,

- relativized to the fragment $\widehat{\mathrm{PA}}^{\omega} \uparrow$ of $\mathcal{A}^{\omega}$, cf. [8, Section 17.1, p. 382] and

- extended by the constant $\left(e_{i}\right)_{i}$ and the purely universal axiom for it, cf. 8 , Section 17.5]

holds.

By Theorem 10]a suitable instance of weak-BW can reduce an instance of $\Sigma_{n+3}^{0}-\mathrm{IA}$ to $\Sigma_{n+1}^{0}$-IA. Thus the system $\widehat{\mathrm{PA}}^{\omega} \uparrow\left[X,\langle\cdot, \cdot\rangle,\left(e_{i}\right)_{i \in \mathbb{N}}\right]+\Sigma_{n+1}^{0}$ IA proves that a suitable instance of weak-BW implies A. Applying the metatheorem to this statement yields terms in $T_{n}$ such that an application of these terms to $\Omega$ yields a solution of the functional interpretation of $\mathrm{A}$.

This prove the corollary.

This shows that Kohlenbach's analysis of $\Omega^{*}$ (a majorant of a solution of the functional interpretation of weak-BW) in [7] is optimal.

This analysis and actually even his proof of weak compactness for abstract Hilbert spaces [9, Theorem 11] shows that only two nested instances of $\Pi_{1}^{0}$-CA (plus some uses of WKL) are needed to proof an instance of weak-BW. Thus, the lower bound on the strength of instances of weak-BW from the Theorems 8 and 10 is strict in the senses that there is no instance of $\Pi_{3}^{0}$-CA which is implied by an instance of weak-BW.

We now give a reversal for the special case of $\ell_{2}$ and analyze the exact computational content:

Theorem 12. For each bounded sequence $\left(x_{i}\right)_{i \in \mathbb{N}}$ in $\ell_{2}$ one can compute uniformly and primitive recursively an function $t$ such that

$$
\mathrm{RCA}_{0} \vdash \Pi_{2}^{0}-\mathrm{CA}(t) \rightarrow \text { weak-BW }\left(\left(x_{i}\right)_{i \in \mathbb{N}}\right) .
$$

In particular, each bounded and computable sequences of $\ell_{2}$ has a weak cluster point computable in $0^{\prime \prime}$.

Proof. We show that provably in $\mathrm{RCA}_{0}$ a cluster point of $\left(x_{i}\right)_{i}$ can be computed in the second Turing jump. The result follows then from the fact that any function computable in the second Turing jump is recursive in a suitable instance of $\Pi_{2}^{0}$-CA.

We assume that $\left(x_{i}\right)_{i}$ is bounded by 1 .

Note that the Bolzano-Weierstraß theorem for the space $[-1,1]^{\mathbb{N}}$ (with the product metric $\left.d\left(\left(x_{i}\right)_{i},\left(y_{i}\right)_{i}\right)=\sum_{i=0}^{\infty} \frac{\min \left(\left|x_{i}-y_{i}\right|, 1\right)}{2^{i+1}}\right)$ is instance-wise equivalent to the Bolzano-Weierstraß theorem for $[-1,1]$. This can easily be seen from the fact that the Bolzano-Weierstraß theorem for $[-1,1]$ is instance-wise equivalent to the theorem for the Cantor space $2^{\mathbb{N}}$ and the fact that $2^{\mathbb{N}}$ is isomorphic to $\left(2^{\mathbb{N}}\right)^{\mathbb{N}}$.

Hence by [10] one can find a cluster point of the sequence

$$
y_{i}:=\left(\left\langle e_{0}, x_{i}\right\rangle,\left\langle e_{1}, x_{i}\right\rangle, \ldots\right)
$$

in $[-1,1]^{\mathbb{N}}$ by computing an infinite path trough a $\Sigma_{1}^{0}$-tree. Call this cluster point $c=\left(c_{0}, c_{1}, \ldots\right) \in[-1,1]^{\mathbb{N}}$.

Claim. $\sum_{j=0}^{\infty} c_{j} \leq 1$ 
Proof of claim. Since the elements of $y_{i}$ are elements of a Hilbert space and are norm bounded by 1 we have that $\sum_{j=0}^{k}\left(y_{i}\right)_{j}^{2} \leq 1$. Now for each $k$ and for each $\varepsilon$ there is an $y_{i}$ such that $\left|c_{j}-\left(y_{i}\right)_{j}\right| \leq \varepsilon$ for $j \leq k$ and hence

$$
\sum_{j=0}^{k}\left(c_{j}\right)^{2} \leq \sum_{j=0}^{k}\left(\left(y_{i}\right)_{j}+\varepsilon\right)^{2} \leq 1+3(k+1) \varepsilon .
$$

From this follows the claim.

Now one easily checks that the sequence $\left(z_{i}\right)_{i \in \mathbb{N}}$ with $z_{i}:=\left\langle c_{0}, \ldots, c_{i}\right\rangle$ converges in the $\ell_{2}$-norm to a weak cluster point $x$ of $\left(x_{i}\right)_{i}$. This convergence is monotone in the sense that $\left\|z_{i}\right\| \leq\left\|z_{i+1}\right\|$ thus the limit point $x$ can be computed in the Turing jump of $\left(z_{i}\right)_{i}$.

The point $x$ is provably computable in the second Turing jump of $\left(x_{i}\right)_{i}$ because $c$ is by the low basis theorem ([6]) computable in a degree provably low over the first Turing jump. (The proof of the low basis theorem is effective and formalizes in $\mathrm{RCA}_{0}$.) Therefore the jump of $\left(z_{i}\right)_{i}$ and thus $x$ is computable in the second Turing jump.

With this we can classify the computational strength of weak compactness on $\ell_{2}$ :

Corollary 13. For a Turing degree $d$ the following are equivalent:

- $d \geq_{T} 0^{\prime \prime}$ and

- $d$ computes a weak cluster point for each computable, bounded sequence in $\ell_{2}$.

As consequence we obtain that the Bolzano-Weierstraß principle for weak compactness is instance-wise strictly stronger than the Bolzano-Weierstraß principle for the unit interval $[0,1]$, cf. [10.

Remark 14 (Weihrauch lattice). The proofs of the Theorems 8 and 12 can also be used to classify the Bolzano-Weierstraß principle for weak compactness in $\ell_{2}$ in the Weihrauch lattice. We do not introduce the notation for the Weihrauch lattice but refer the reader to 3 .

Let $\mathrm{BWT}_{\text {weak }-\ell_{2}}: \subseteq\left(\ell_{2}\right)^{\mathbb{N}} \rightrightarrows \ell_{2}$ be the partial multifunction which maps bounded sequences of $\ell_{2}$ to a weak cluster point of that sequence.

The proof of Theorem 8 immediately yields that

$$
\mathrm{BWT}_{\text {weak }-\ell_{2}} \geq_{\mathrm{W}} \widehat{\mathrm{LPO}} \circ \widehat{\mathrm{LPO}} \equiv_{\mathrm{W}} \lim ^{(2)} \text {. }
$$

Whereas the proof of Theorem 8 yields that

$$
\mathrm{BWT}_{\text {weak- } \ell_{2}} \leq_{\mathrm{W}} \mathrm{MCT} * \mathrm{BWT}_{\mathbb{R}^{\mathbb{N}}} .
$$

The function $\mathrm{BWT}_{\mathbb{R}^{\mathbb{N}}}$ is used to compute the cluster point $c \in \mathbb{R}^{\mathbb{N}}$, the function MCT is used for the convergence of $\left(\left\|z_{i}\right\|\right)_{i}$. By the same argument as in the proof $B W T_{\mathbb{R}} \equiv_{W} B W T_{\mathbb{R}^{N}}$. Since all of these multifunctions are cylinders one may also strengthen the reducibility to strong Weihrauch reducibility. Thus

$$
\begin{aligned}
\mathrm{BWT}_{\text {weak }-\ell_{2}} & \leq_{\mathrm{sW}} \mathrm{MCT} *_{\mathrm{s}} \mathrm{BWT}_{\mathbb{R}} \\
& \leq_{\mathrm{sW}} \lim *_{\mathrm{s}} \mathfrak{L}^{\prime} \\
& \leq_{\mathrm{sW}} \lim *_{\mathrm{s}} \mathfrak{L}_{1,2} \\
& \equiv_{\mathrm{sW}} \lim \circ \lim .
\end{aligned}
$$


(For the last equivalence see [3, Corollary 8.8], which is a consequence of an analysis of the low basis theorem in the Weihrauch lattice, see [2.)

In total we obtain that

$$
\text { BWT }_{\text {weak- } \ell_{2}} \equiv_{\mathrm{sW}} \lim ^{(2)} \text {. }
$$

As consequence we also obtain that $\mathrm{BWT}_{\text {weak } \ell_{2}}>_{\mathrm{sW}} \mathrm{BWT}_{\mathbb{R}}$.

\section{REFERENCES}

1. Jeremy Avigad and Ksenija Simic, Fundamental notions of analysis in subsystems of secondorder arithmetic, Ann. Pure Appl. Logic 139 (2006), no. 1-3, 138-184. MR 2206254

2. Vasco Brattka, Matthew de Brecht, and Arno Pauly, Closed Choice and a Uniform Low Basis Theorem, preprint at http://arxiv.org/abs/1002.2800

3. Vasco Brattka, Guido Gherardi, and Alberto Marcone, The Bolzano-Weierstrass Theorem is the Jump of Weak König's Lemma, preprint at http://arxiv.org/abs/1101.0792

4. Philipp Gerhardy and Ulrich Kohlenbach, General logical metatheorems for functional analysis, Trans. Amer. Math. Soc. 360 (2008), no. 5, 2615-2660. MR 2373327

5. Petr Hájek and Pavel Pudlák, Metamathematics of first-order arithmetic, Perspectives in Mathematical Logic, Springer-Verlag, Berlin, 1998, Second printing. MR 1748522

6. Carl G. Jockusch, Jr. and Robert I. Soare, $\Pi_{1}^{0}$ classes and degrees of theories, Trans. Amer. Math. Soc. 173 (1972), 33-56. MR 0316227

7. Ulrich Kohlenbach, Gödel functional interpretation and weak compactness, accepted for publication in Ann. Pure Applied Logic, preprint at http://www.mathematik.tu-darmstadt.de/ kohlenbach/

8. - Applied proof theory: Proof interpretations and their use in mathematics, Springer Monographs in Mathematics, Springer Verlag, 2008. MR 2445721

9. - On the logical analysis of proofs based on nonseparable Hilbert space theory, Proofs, Categories and Computations. Essays in Honor of Grigori Mints (S. Feferman and W. Sieg, eds.), College Publications, 2010.

10. Alexander P. Kreuzer, The Bolzano-Weierstraß principle and the cohesive principle, Math. Logic Quart. 57 (2011), no. 3, 292-298.

11. Stephen G. Simpson, Subsystems of second order arithmetic, Perspectives in Mathematical Logic, Springer-Verlag, Berlin, 1999. MR 1723993

Fachbereich Mathematik, Technische Universität Darmstadt, Schlossgartenstrasse 7 , 64289 Darmstadt, Germany

E-mail address: akreuzer@mathematik.tu-darmstadt.de 